\title{
Guest editorial: revised selected papers from the LION 8 conference
}

\author{
Panos M. Pardalos ${ }^{1}$. Chrysafis Vogiatzis ${ }^{2}$. \\ Jose L. Walteros ${ }^{3}$
}

Published online: 9 October 2015

(C) Springer International Publishing Switzerland 2015

In February 2014, we had the honor of organizing the eighth installment of the conference series "Learning and Intelligent Optimization" (LION) in Gainesville, Florida, USA. As always, the work presented was at the forefront of research performed between the fields of Artificial Intelligence, Mathematical Programing and Optimization, and Algorithmic Design. The conference was very successful in attracting researchers from around the world who have been pushing the boundaries in the intersection of the above fields, among others.

As has become tradition from the earlier, very successful LION conferences, we are happy to dedicate this special issue to extended versions of a selected sample of the work presented in LION 8 in February 2014. For the remainder of the contributions submitted and presented during LION 8, we refer the interested reader to [1].

The spectrum of the topics covered in this special issue ranges from facility location and routing, to job scheduling and robotics, while the techniques and approaches come from fields as diverse as algorithm portfolio techniques, evolutionary algorithms, and hybrid metaheuristics. More specifically this special issue contains the following contributions.

The first paper, Bayesian Optimization for Learning Gaits Under Uncertainty by Roberto Calandra, André Seyfarth, Jan Peters, and Marc Peter Deisenroth, deals with the problem

Panos M. Pardalos

pardalos@ise.ufl.edu

Chrysafis Vogiatzis

chrysafis.vogiatzis@ndsu.edu

Jose L. Walteros

josewalt@buffalo.edu

1 Department of Industrial and Systems Engineering, University of Florida, Gainesville, FL, USA

2 Department of Industrial and Manufacturing Engineering, North Dakota State University, Fargo, ND, USA

3 Department of Industrial and Systems Engineering, State University of New York at Buffalo, Buffalo, NY, USA 
of identifying the gait parameters that optimize the robustness and energy consumption required for robotic bipedal locomotion.

Then, the second article, Controlling Selection Areas of Useful Infeasible Solutions for Directed Mating in Evolutionary Constrained Multi-Objective Optimization by Minami Miyakawa, Keiki Takadama, and Hiroyuki Sato proposes a method that restricts the areas from which an evolutionary algorithm selects infeasible, but possibly good candidate solutions, in order to improve the performance of directed mating when solving constrained multi-objective optimization problems.

The third contribution, entitled On Some Statistical Procedures for Stock Selection Problem by Petr Koldanov, Valeriy Kalyagin, and Grigory Bautin discusses the problem of stock selection and possible statistical analysis procedures to tackle it.

The paper Improving Local-Search Metaheuristics Through Look-Ahead Policies by David Meignan, Stefan Voß, and Silvia Schwarze discusses local search metaheuristics and the implementation of "look-ahead" policies for improving the quality of solution obtained.

The fifth paper presented here, On Multivariate Network Analysis of Statistical Data Sets with Different Measures of Association by Valeriy Kalyagin, Alexander Koldanov, and Panos M. Pardalos, investigates different association measures for use with network analysis of statistical datasets.

The sixth paper of the special issue, Models and Algorithms for Competitive Facility Location Problems with Different Customer Behavior by Benjamin Biesinger, Bin Hy, and Günther Raidl, discusses competitive facility location under a bi-level optimization framework.

Continuing, the paper A Hybrid Clonal Selection Algorithm for the Location Routing Problem with Stochastic Demands by Yannis Marinakis, Magdalene Marinaki, and Athanasios Migdalas, discusses a clonal selection algorithm variant and its application to the capacitated location routing problem under uncertainty.

The eight article, Algorithm Portfolios for Noisy Optimization by Marie-Liesse Cauwet, Jialin Liu, Baptiste Roziére, and Olivier Teytaud, studies portfolios of noisy optimization solvers, and investigates their performance,

Furthermore, the article POPMUSIC as a Matheuristic for the Berth Allocation Problem by Eduardo Laila-Ruiz and Stefan Voß, proposes a novel metahueristic approach, which is a hybrid of traditional metaheuristics and mathematical programming techniques, and shows its performance in the berth allocation problem.

The tenth contribution, GeneRa: A Problem Generator for Radiotherapy Treatment Scheduling Problems by Maria-Cristina Riff, Juan Pablo Cares, and Bertrand Neveu attempts to address the lack of configurable benchmark generators for radiotherapy scheduling in the literature.

Next we have a manuscript entitled Scheduling Jobs on a Single Serial-Batching Machine with Dynamic Job Arrivals and Multiple Job Types by Jun Pei, Xinbao Liue, Wenjuan Fan, Panos M. Pardalos, Athanasios Migdalas, and Shanlin Yang, deals with job scheduling under dynamic job arrival and for multiple job types.

Last, the paper by Roberto Amadini, Maurizio Gabbrielli, and Jacopo Mauro titled Portfolio Approaches for Constraint Optimization Problems, provides strong evidence that some portfolio approaches that are commonly used to solve constraint satisfiability problems can also be successful when tackling difficult instances of combinatorial optimization problems. In this paper, the authors compare three different portfolio techniques, the first one is adapted from the satisfiability world, the second one is based on Machine Learning classifiers, and the third one is a lazy portfolio selection approach. 
We would like to thank the authors for contributing their work, the reviewers who made certain the quality of the contributions remains at the highest standards, and, of course, the researchers and practitioners who attended the LION 8 conference. It is because of these people that our field is continuing its growth and is aiming to chart the unexplored areas and consolidate novel knowledge and practice approaches.

\section{References}

1. Pardalos, P.M., Resende, M.G., Vogiatzis, C., Walteros, J.L.: Learning and intelligent optimization. In: 8th International Conference, Lion 8. Gainesville. Revised Selected Papers, vol. 8426. Springer (2014) (2014) 